\title{
Mesenchymal Stem Cell Marker Expression in Gastric Cancer Stroma
}

\author{
SATOE NUMAKURA, HIROSHI UOZAKI, YOSHINAO KIKUCHI, \\ SHIORI WATABE, ARISA TOGASHI and MASATO WATANABE
}

Department of Pathology, Teikyo University School of Medicine, Tokyo, Japan

\begin{abstract}
Background/Aim: Spindle-shaped stromal cells of tumors are derived from various cellular origins, including mesenchymal stem cells (MSCs). MSCs express CD73, CD90 and CD105 antigens. Herein, the aim was to investigate the association between the expression of specific MSC markers in gastric cancer stromal cells and the clinicopathological features of the disease. Materials and Methods: The expression of CD73, CD90 and CD105 in spindle-shaped cancer stromal cells was studied by immunohistochemistry in tissue arrays containing 546 gastric cancer cases. Univariate and multivariate analyses were performed to evaluate the association of MSC marker expression with clinicopathological variables. Results: Spindle-shaped cancer stromal cells expressing the MSC markers CD73, CD90 or CD105 were associated with larger tumor size, advanced cancer, venous infiltration, lymphatic infiltration, and lymph node metastasis. Statistical analysis demonstrated that the presence of CD105positive spindle cells was an independent prognostic factor of advanced cancer, lymph node metastasis and EBV infection in multivariate analysis. Conclusion: Spindle-shaped gastric cancer stromal cells expressing CD73, CD90 or CD105 are involved in disease progression, and among them, CD105positive cells are strongly associated with poor prognosis.
\end{abstract}

The prognosis of advanced gastric cancer is still poor $(1,2)$ and identifying the mechanism of cancer progression remains challenging (2). It is well known that interactions between carcinoma cells and the tumor stroma are crucial for tumor growth and progression $(3,4)$. Among cancer stromal cells, research has largely focused on cancer-associated fibroblasts (CAFs); however, their study is hampered due to the lack of

Correspondence to: Hiroshi Uozaki, Department of Pathology, Teikyo University School of Medicine, 2-11-1 Kaga, Itabashi-ku, Tokyo 173-8606, Japan. Tel: +81 339643498, e-mail: uozaki@med.teikyo-u.ac.jp

Key Words: Gastric cancer, cancer stroma, mesenchymal stem cell, immunohistochemistry. specific markers to identify CAFs (3). CAFs comprise a heterogeneous population of cells with various origins, including resident fibroblasts, fibrocytes, pericytes, endothelial cells, smooth muscle cells, adipocytes, epithelial cells, bone marrow-derived cell and mesenchymal stem cells (MSCs) (3-5). The latter MSCs seem to play a critical role in cancer progression (6).

Multipotent MSCs are spindle-shaped and are found at perivascular sites in various human tissues and organs $(7,8)$. MSCs are introduced to a tumor microenvironment by soluble factors released from inflammatory cells or tumor cells (9). MSCs, as defined by the International Society for Cellular Therapy (ISCT), express CD73 (Ecto-5'-nucleotidase), CD90 (Thy-1) and CD105 (Endoglin) (10). Previous cancer studies used MSCs mainly isolated from bone marrow or umbilical cord blood (11-14). MSC-like cells isolated from human gastric cancer stroma (GC-MSCs) were found to possess similar characteristics to bone marrow-derived MSCs (BMMSCs), including spindle-shaped morphology and MSC immunophenotype (15). However, the two cell populations differed in the number of cytoplasmic organelles and the higher growth ability and long-term proliferation of GCMSCs compared to BM-MSCs (16). In gastric cancer, MSCs that come in contact with premalignant or malignant epithelial cells enhance growth and promote epithelial to mesenchymal transition, thus promoting cancer development and progression (6); whereas gastric cancer cells can also trigger MSCs to differentiate into CAFs (17).

To our knowledge, this is the first study to investigate the association of the expression of MSC markers with clinicopathological factors in a large number of gastric cancer cases. The expression of CD73, CD90 or CD105 in spindle-shaped gastric cancer stromal cells was assessed, and their association with cancer progression was examined.

\section{Patients and Methods}

Tissue arrays. A total of 546 primary gastric cancers from 524 patients surgically resected from 2000 to 2011 were obtained from the archives of the Department of Pathology, Teikyo University 
Hospital, Tokyo, Japan. Some of the gastric cancer cases were included in our previous report (18). This study received approval from the Ethics Committee of the Teikyo University School of Medicine (No. 13-021, 8 May 2013). Formalin-fixed, paraffinembedded (FFPE) tissue blocks were used for staining. Histopathological data were evaluated according to the Japanese classification of gastric cancer and the Lauren classification. The clinicopathological data of the patients and tumors are summarized in Table I. Cylindrical tissue cores of $2 \mathrm{~mm}$ from the primary FFPE blocks were arrayed by using the manual device KIN-1 (Azumaya, Tokyo, Japan). Two representative cores were taken from each tissue block. The resulting tissue array blocks were then sectioned at $4 \mu \mathrm{m}$ in thickness and placed on slides. Cancers occupying more than half of the core area were evaluated by immunohistochemistry.

Immunohistochemical staining. Paraffin sections were dewaxed in xylene and rehydrated through graded ethanol to distilled water. Tissue arrays were stained with rabbit polyclonal antibodies against human CD73 (HPA017357, 1:5000, Atlas Antibodies, Stockholm, Sweden), human CD90 (HPA003733, 1:100, Atlas Antibodies, Stockholm, Sweden) and human CD105 (HPA011862, 1:500, Sigma-Aldrich, St. Louis, USA) using the EnVision FLEX system (Agilent, Santa Clara, USA). Antigen retrieval was performed via water bath heating for $40 \mathrm{~min}$ in retrieval buffers (pH9 for CD73 and CD105, pH6 for CD90). Positive staining was scored only in spindle cells. Since endothelial cells are also spindle-shaped, spindle cells forming a lumen structure were excluded from scoring (19). Scoring scale was as follows (Figure 1): for CD73, according to the positivity rate in a high power field; 0 [0-10\%], 1 [10-50\%], 2 [50$100 \%$ ]. For CD90 and CD105, 0 [absent], 1 [positive in small aggregates], 2 [positive in diffuse fashion], 3 [highly positive area in more than half of core field]. Scoring was performed by two independent pathologists. In case of discordance, a consensus was reached through discussion. After scoring, gastric cancer cases were divided into negative- (score 0 ) and positive- (score $\geq 1$ ) expression groups for CD73, CD90 and CD105. Epstein-Barr virus (EBV) infection was evaluated by EBER in situ hybridization as previously described (18).

Statistical analyses. Chi-square test was used to determine whether there was significant association between MSC marker expression and clinicopathological features. The $t$-test was used for comparisons between two samples from the same population after stratification. Survival curves were estimated by the Kaplan-Meier method and compared by the log-rank test. Multivariate analyses were performed using Cox proportional hazards model. For the latter, scoring data of miR-21 expression and EBER in situ hybridization from our previous report were used (18). All $p$-values of less than 0.05 were regarded as significant.

\section{Results}

Expression of MSC markers in spindle-shaped gastric cancer stroma cells. All markers showed membranous staining, while CD73 and CD90 were also found in the cytoplasm. The expression of CD73, CD90 and CD105 was detected in mononuclear cells and, in some cases, in vascular endothelial cells, and spindle-shaped cells; CD73 was also expressed in
Table I. Patients and gastric cancer cases characteristics.

\begin{tabular}{lc}
\hline Characteristic & $\mathrm{n}$ \\
\hline Patients & 524 \\
Tumor cases & 546 \\
Age (years) & $67.8 \pm 10.9^{*}$ \\
Gender (male/female) & $373 / 151$ \\
Tumor size (mm) & $55.1 \pm 50.7^{*}$ \\
Tumor stage (early/advanced) ${ }^{\dagger}$ & $207 / 317$ \\
Lauren classification (intestinal/diffuse) & $286 / 238$ \\
Lymphatic infiltration (negative/positive) & $247 / 277$ \\
Venous infiltration (negative/positive) & $250 / 274$ \\
Lymph node metastasis (negative/positive) & $258 / 266$ \\
EBV (negative/positive) & $485 / 39$ \\
\hline
\end{tabular}

*Mean \pm standard deviation. ${ }^{\dagger}$ Early=Tis or $\mathrm{T} 1$, in TNM classification.

Table II. Distribution of immunostaining scores in spindle cells.

\begin{tabular}{lrrr}
\hline Score & CD73 & CD90 & CD105 \\
\hline 0 & 356 & 213 & 485 \\
1 & 93 & 192 & 59 \\
2 & 97 & 122 & 2 \\
3 & - & 19 & 0 \\
\hline
\end{tabular}

Table III. Correlation between the expressions of the examined MSC markers.

\begin{tabular}{|c|c|c|c|c|c|c|}
\hline & \multicolumn{3}{|c|}{ CD73 } & \multicolumn{3}{|c|}{ CD90 } \\
\hline & Negative & Positive & $p$-Value & Negative & Positive & $p$-Value \\
\hline \multicolumn{7}{|l|}{ CD90 } \\
\hline Negative & 188 & 25 & $<0.0001$ & & & \\
\hline Positive & 168 & 165 & & & & \\
\hline \multicolumn{7}{|l|}{ CD105 } \\
\hline Negative & 330 & 155 & 0.0002 & 208 & 277 & $<0.0001$ \\
\hline Positive & 26 & 35 & & 5 & 56 & \\
\hline
\end{tabular}

cancer cells. In gastric cancer stroma, 190 (34.8\%) cases were positive for CD73, $333(61.0 \%)$ cases for CD90 and 61 (11.2\%) cases for CD105 (Table II). In addition, there was a positive correlation among the expression of CD73, CD90 and CD105 $(p<0.0001$ for CD73 and CD90, $p=0.0002$ for CD73 and CD105, and $p<0.0001$ for CD90 and CD105) (Table III).

MSC marker expression was related to disease progression. The association of each MSC marker with clinico- 


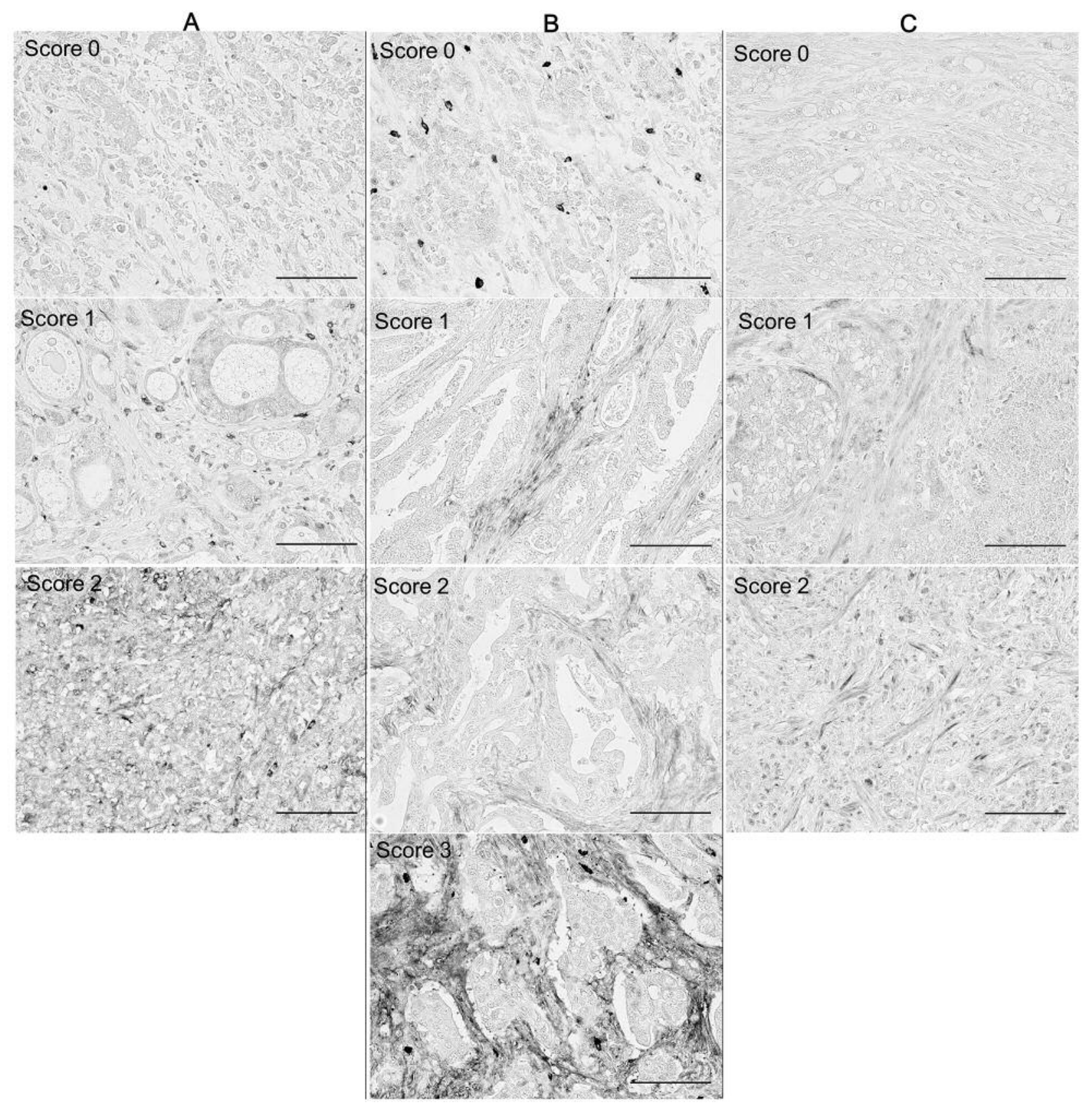

Figure 1. Immunohistochemical assessment of the expression of each marker in spindle cells of gastric cancer stroma. Representative cases are shown; CD73 (A: score 0-2), CD90 (B: score 0-3), CD105 (C: score 0-2). Scale bar=100 $\mu \mathrm{m}$.

pathological features was examined (Table IV). The expression of all markers was positively correlated with larger tumor size $(p=0.0001$ for CD73, $p<0.0001$ for CD90, $p=0.0009$ for CD105), advanced cancer $(p<0.0001$ for CD73, CD90 and CD105), venous infiltration $(p<0.0001$ for CD73 and CD90, $p=0.001$ for CD105), lymphatic infiltration $(p<0.0001$ for CD73, CD90 and CD105) and lymph node metastasis $(p<0.0001$ for CD73 and CD90, $p=0.0002$ for CD105). Concerning CD73 and CD90 markers, positivity was also associated with diffuse type in Lauren's classification ( $p=0.009$ for CD73, $p=0.0002$ for CD90). These results suggest that the expression of the examined MSC markers in gastric cancer stroma is associated with disease progression. 
Table IV. Clinicopathological features and MSC markers.

\begin{tabular}{|c|c|c|c|c|c|c|c|c|c|}
\hline & \multicolumn{3}{|c|}{ CD73 } & \multicolumn{3}{|c|}{ CD90 } & \multicolumn{3}{|c|}{ CD105 } \\
\hline & Negative & Positive & $p$-Value & Negative & Positive & $p$-Value & Negative & Positive & $p$-Value \\
\hline \multicolumn{10}{|l|}{ Gender } \\
\hline Male & 258 & 134 & 0.690 & 154 & 238 & 0.846 & 349 & 43 & 0.88 \\
\hline Female & 98 & 56 & & 59 & 95 & & 136 & 18 & \\
\hline Age (years) & $67.3 \pm 11.4^{\dagger}$ & $69.0 \pm 10.0$ & 0.075 & $67.8 \pm 11.5$ & $68.0 \pm 10.6$ & 0.799 & $67.6 \pm 11.2$ & $70.0 \pm 8.5$ & 0.115 \\
\hline Size $(\mathrm{mm})$ & $47.8 \pm 54.6^{\dagger}$ & $65.3 \pm 38.4$ & 0.0001 & $38.0 \pm 36.5$ & $63.9 \pm 55.1$ & $<0.0001$ & $51.2 \pm 50.2$ & $73.9 \pm 46.7$ & 0.0009 \\
\hline \multicolumn{10}{|l|}{ Stage } \\
\hline Early & 200 & 27 & $<0.0001$ & 153 & 74 & $<0.0001$ & 220 & 7 & $<0.0001$ \\
\hline Advanced & 156 & 163 & & 60 & 259 & & 265 & 54 & \\
\hline \multicolumn{10}{|c|}{ Venous infiltration } \\
\hline Negative & 218 & 53 & $<0.0001$ & 166 & 105 & $<0.0001$ & 253 & 18 & 0.001 \\
\hline Positive & 138 & 137 & & 47 & 228 & & 232 & 43 & \\
\hline \multicolumn{10}{|c|}{ Lymphatic infiltration } \\
\hline Negative & 213 & 53 & $<0.0001$ & 157 & 109 & $<0.0001$ & 252 & 14 & $<0.0001$ \\
\hline Positive & 143 & 137 & & 56 & 224 & & 233 & 47 & \\
\hline \multicolumn{10}{|c|}{ Lymph node metastasis } \\
\hline Negative & 228 & 49 & $<0.0001$ & 163 & 114 & $<0.0001$ & 260 & 17 & 0.0002 \\
\hline Positive & 128 & 141 & & 50 & 219 & & 225 & 44 & \\
\hline \multicolumn{10}{|c|}{ Lauren's classification } \\
\hline Diffuse & 143 & 99 & 0.009 & 73 & 169 & 0.0002 & 210 & 32 & 0.218 \\
\hline Intestinal & 213 & 91 & & 140 & 164 & & 275 & 29 & \\
\hline \multicolumn{10}{|l|}{ EBV status } \\
\hline Negative & 322 & 183 & 0.016 & 199 & 306 & 0.618 & 449 & 56 & 0.797 \\
\hline Positive & 34 & 7 & & 14 & 27 & & 36 & 5 & \\
\hline
\end{tabular}

$\dagger$ Mean \pm standard deviation.

MSC markers and prognosis. The expression of CD73, CD90 and CD105 was associated with poorer prognosis $(p<0.0001$ for CD73 and CD105, $p=0.0001$ for CD90) (Figure 2). A multivariate analysis was performed that included CD73-positive spindle cells, CD90-positive spindle cells, CD105-positive spindle cells, stage, venous infiltration, lymphatic infiltration, lymph node metastasis, and miR-21 expression in cancer stroma (previous data (18)) and EBV status (previous data (18)). CD105-positive spindle cells were strongly associated with poor prognosis (Adjusted risk ratio $=2.407,95 \% \mathrm{CI}=1.463-3.961, p=0.0005)($ Table V).

\section{Discussion}

Previous studies indicated that the interaction between cancer cells and MSCs is involved in the epithelial to mesenchymal transition and in the differentiation of MSCs to CAFs, as well $(6,17)$. Hence, this interaction is a critical event in gastric cancer progression. To investigate the possible association of MSC features with gastric cancer progression, the correlation between the expression of three MSC markers, CD73, CD90 and $\mathrm{CD} 105$, in spindle-shaped stromal cells, and clinicopathological features was examined in a large number of cases. We found that the expression of these markers was associated with larger tumor size, advanced stage, venous infiltration, lymphatic infiltration and lymph node metastasis. These data suggested the expression of MSC markers was associated with disease progression.

There are several reports regarding the relationship between the tested MSC markers and cancer. Contrary to our results, CD73 expression in cancer stroma was associated with better prognosis in rectal cancer (20) and prostate cancer (21). This could be attributed to the possibility that the stromal component included round immune cells in these reports. However, this discrepancy needs to be further explored. CD90 can be expressed in the cancer stroma of prostate (22), lung (23) and gastric cancer (24). In lung cancer, it was that suggested CD90 is a CAF marker (23). CD90-positive CAFs affect the prostate cancer stem cell niche (22) and promote lung cancer cell proliferation and dissemination (25). Moreover, in vivo studies in mice and in vitro experiments in human prostate-derived CAFs have demonstrated that CD105 expression was also involved in prostate cancer progression (26). Authors suggested that CD105-positive fibroblasts were derived from myofibroblasts rather than from resident fibroblasts (26).

A recent report, based on human clinical samples, showed that high percentage of triple-positive $\left(\mathrm{CD} 73^{+} / \mathrm{CD}^{+} 0^{+}\right.$ $\mathrm{CD}_{105^{+}}$) stromal cells from high-grade glioma tissue 

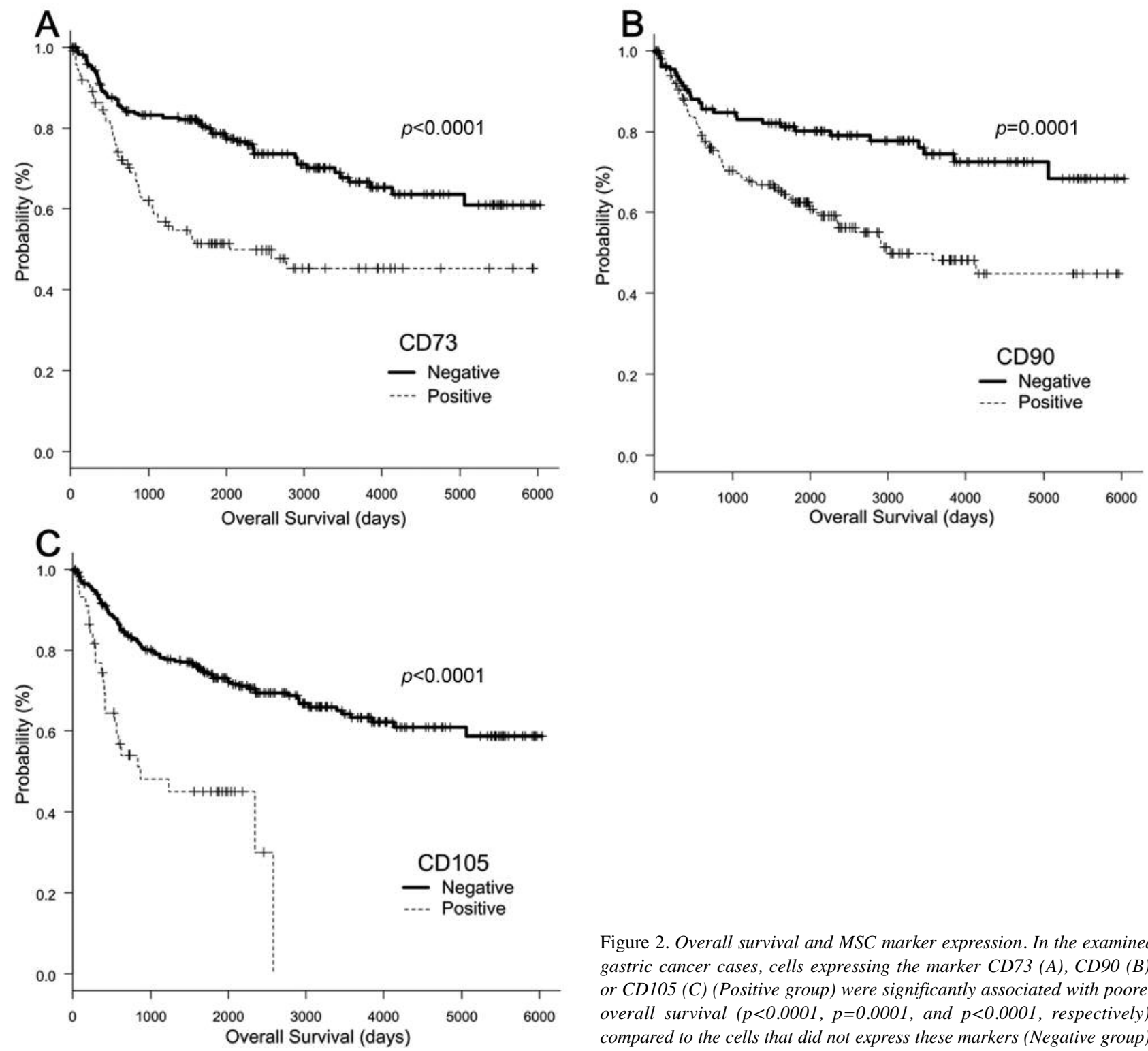

Figure 2. Overall survival and MSC marker expression. In the examined gastric cancer cases, cells expressing the marker CD73 (A), CD90 (B), or CD105 (C) (Positive group) were significantly associated with poorer overall survival $(p<0.0001, p=0.0001$, and $p<0.0001$, respectively), compared to the cells that did not express these markers (Negative group).

Table V. Prognostic factors for patient survival in univariate and multivariate analyses.

\begin{tabular}{lcrcr}
\hline Characteristics & Unadjusted risk ratio $(95 \% \mathrm{CI})$ & $p$-Value & Adjusted risk ratio $(95 \% \mathrm{CI})$ & $p$-Value \\
\hline $\mathrm{CD}^{+} 3^{+}$spindle cells & $2.251(1.554-3.262)$ & $<0.0001$ & $0.908(0.597-1.383)$ & 0.6540 \\
$\mathrm{CD}^{+}$spindle cells & $2.202(1.461-3.320)$ & 0.0002 & $0.669(0.403-1.111)$ & 0.1205 \\
$\mathrm{CD}^{+} 5^{+}$spindle cells & $3.153(1.986-5.004)$ & $<0.0001$ & $2.407(1.463-3.961)$ & 0.0005 \\
miR-21 expression & $1.747(1.214-2.515)$ & 0.0027 & $1.020(0.694-1.499)$ & 0.9195 \\
Advanced stage & $7.013(4.211-11.68)$ & $<0.0001$ & $3.745(1.814-7.735)$ & 0.0004 \\
Lymphatic infiltration & $3.790(2.522-5.694)$ & $<0.0001$ & $1.173(0.690-1.994)$ & 0.5559 \\
Venous infiltration & $3.657(2.450-5.457)$ & $<0.0001$ & $1.270(0.772-2.089)$ & 0.3471 \\
Lymph node metastasis & $4.508(2.979-6.821)$ & $<0.0001$ & $2.208(1.266-3.853)$ & 0.0053 \\
Positive EBV status & $0.399(0.175-0.908)$ & 0.0285 & $0.434(0.190-0.994)$ & 0.0484 \\
\hline
\end{tabular}

CI, Confidence interval; EBV, Epstein-Barr virus. 
(Glioma-associated MSCs) were correlated with poor prognosis (27). Herein, co-expression of CD73, CD90, and CD105 was not examined, but a positive correlation among them was revealed. According to multivariate analysis, among the three MSC markers, CD105 was the most important prognostic factor of overall survival of gastric cancer. This predominance of CD105 over other MSC markers was also suggested in multipotency, one of the essential traits of MSCs, where $\mathrm{CD}^{2} 3^{-} / \mathrm{CD}_{105^{+}}$dermal fibroblasts showed differentiation into both adipocytes and osteocytes, whereas $\mathrm{CD}^{2} 3^{-} / \mathrm{CD} 105^{-}$dermal fibroblasts did not (28). This difference among markers could be partly explained from the close relationship between CD105 and TGF-beta signaling. CD105 is a co-receptor of TGF-beta, which directly modulates signaling (29). This process is necessary for angiogenesis and vasculogenesis in endothelial cells or immature mesenchymal cells, as well as for fibrosis in fibroblasts in benign and malignant states (30). In the human stomach, TGF-beta 1 was expressed in epithelial cells and cancer cells closely associated with CD105-positive neo-vessels (31). High density of CD105-positive micro-vessels (neo-vessels) was associated with poorer prognosis in several types of cancer (29).

Since endothelial cells are spindle-shaped (3), we defined "spindle cells" as cells with a spindle-shaped morphology without lumen formation (19). However, there is a possibility that "spindle cells" included the endothelium of collapsed or immature vessels with obscured lumens. On the other hand, it is also possible that aggregates of CD105-positive spindle cells were immature mesenchymal cells associated with vasculogenesis.

Our study showed that CD105-positive spindle stromal cells are strongly associated with disease progression and poor prognosis of gastric cancer, suggesting that these cells may represent a promising therapeutic target for gastric cancer.

\section{Acknowledgements}

This work was supported by our host institutions. The Authors declare no conflicts of interest.

\section{References}

1 Van Cutsem E, Sagaert X, Topal B, Haustermans K and Prenen H: Gastric cancer. Lancet 388: 2654-2664, 2016.

2 Aso T, Uozaki H, Morita S, Kumagai A and Watanabe M: Loss of ARID1A, ARID1B, and ARID2 expression during progression of gastric cancer. Anticancer Res 35: 6819-6827, 2015.

3 Delinasios JG, Angeli F, Koumakis G, Kumar S, Kang WH, Sica G, Iacopino F, Lama G, Lamprecht S, Sigal-Batikoff I, Tsangaris GT, Farfarelos CD, Farfarelos MC, Vairaktaris E, Vassiliou S and Delinasios GJ: Proliferating fibroblasts and HeLa cells cocultured in vitro reciprocally influence growth patterns, protein expression, chromatin features and cell survival. Anticancer Res 35: 1881-1916, 2015.
4 Shiga K, Hara M, Nagasaki T, Sato T, Takahashi H and Takeyama H: Cancer-associated fibroblasts: Their characteristics and their roles in tumor growth. Cancers (Basel) 7: 2443-2458, 2015.

5 Yan Y, Wang L-F and Wang R-F: Role of cancer-associated fibroblasts in invasion and metastasis of gastric cancer. World $\mathrm{J}$ Gastroenterol 21: 9717-9726, 2015.

6 Xue J, Zhu Y, Sun Z, Ji R, Zhang X, Xu W, Yuan X, Zhang B, Yan Y, Yin L, Xu H, Zhang L, Zhu W and Qian H: Tumorigenic hybrids between mesenchymal stem cells and gastric cancer cells enhanced cancer proliferation, migration and stemness. BMC Cancer 15: 793, 2015.

7 Melzer C, von der Ohe J, Lehnert H, Ungefroren H and Hass R: Cancer stem cell niche models and contribution by mesenchymal stroma/stem cells. Mol Cancer 16: 28, 2017.

8 Hass R, Kasper C, Böhm S and Jacobs R: Different populations and sources of human mesenchymal stem cells (MSC): A comparison of adult and neonatal tissue-derived MSC. Cell Commun Signal 9: 12, 2011.

9 Gomes CMF: The dual role of mesenchymal stem cells in tumor progression. Stem Cell Res Ther 4: 42, 2013.

10 Dominici M, Le Blanc K, Mueller I, Slaper-Cortenbach I, Marini FC, Krause DS, Deans RJ, Keating A, Prockop DJ and Horwitz EM: Minimal criteria for defining multipotent mesenchymal stromal cells. The International Society for Cellular Therapy position statement. Cytotherapy 8: 315-317, 2006.

11 Ishii G, Sangai T, Oda T, Aoyagi Y, Hasebe T, Kanomata N, Endoh Y, Okumura C, Okuhara Y, Magae J, Emura M, Ochiya $\mathrm{T}$ and Ochiai A: Bone-marrow-derived myofibroblasts contribute to the cancer-induced stromal reaction. Biochem Biophys Res Commun 309: 232-240, 2003.

12 Zhu W, Xu W, Jiang R, Qian H, Chen M, Hu J, Cao W, Han C and Chen Y: Mesenchymal stem cells derived from bone marrow favor tumor cell growth in vivo. Exp Mol Pathol 80: 267-274, 2006.

13 Zhu W, Huang L, Li Y, Zhang X, Gu J, Yan Y, Xu X, Wang M, Qian $\mathrm{H}$ and $\mathrm{Xu} \mathrm{W}$ : Exosomes derived from human bone marrow mesenchymal stem cells promote tumor growth in vivo. Cancer Lett 315: 28-37, 2012.

14 Zhu W, Huang L, Li Y, Qian H, Shan X, Yan Y, Mao F, Wu X and $\mathrm{Xu}$ WR: Mesenchymal stem cell-secreted soluble signaling molecules potentiate tumor growth. Cell Cycle 10: 3198-3207, 2011.

$15 \mathrm{Xu}$ X, Zhang X, Wang S, Qian H, Zhu W, Cao H, Wang M, Chen $\mathrm{Y}$ and $\mathrm{Xu} \mathrm{W}$ : Isolation and comparison of mesenchymal stem-like cells from human gastric cancer and adjacent noncancerous tissues. J Cancer Res Clin Oncol 137: 495-504, 2011.

16 Cao H, Xu W, Qian H, Zhu W, Yan Y, Zhou H, Zhang X, Xu X, Li J, Chen Z and Xu X: Mesenchymal stem cell-like cells derived from human gastric cancer tissues. Cancer Lett 274: 6171, 2009.

17 Gu J, Qian H, Shen L, Zhang X, Zhu W, Huang L, Yan Y, Mao F, Zhao C, Shi Y and Xu W: Gastric cancer exosomes trigger differentiation of umbilical cord derived mesenchymal stem cells to carcinoma-associated fibroblasts through TGF- $\beta / \mathrm{Smad}$ pathway. PLoS One 7: e52465, 2012.

18 Uozaki H, Morita S, Kumagai A, Aso T, Soejima Y, Takahashi Y and Fukusato T: Stromal miR-21 is more important than miR21 of tumour cells for the progression of gastric cancer. Histopathology 65: 775-783, 2014. 
19 DeCicco-Skinner KL, Henry GH, Cataisson C, Tabib T, Gwilliam JC, Watson NJ, Bullwinkle EM, Falkenburg L, O'Neill RC, Morin A and Wiest JS: Endothelial cell tube formation assay for the in vitro study of angiogenesis. J Vis Exp 91: e51312, 2014.

20 Zhang B, Song B, Wang X, Chang XS, Pang T, Zhang X, Yin K and Fang GE: The expression and clinical significance of CD73 molecule in human rectal adenocarcinoma. Tumor Biol 36: 5459-5466, 2015.

21 Leclerc BG, Charlebois R, Chouinard G, Allard B, Pommey S, Saad F and Stagg J: CD73 expression is an independent prognostic factor in prostate cancer. Clin Cancer Res 22: 15866, 2016.

22 Pascal LE, Ai J, Vêncio RZN, Vêncio EF, Zhou Y, Page LS, True LD, Wang $\mathrm{Z}$ and Liu AY: Differential inductive signaling of $\mathrm{CD} 90^{+}$prostate cancer-associated fibroblasts compared to normal tissue stromal mesenchyme cells. Cancer Microenviron 4: 51-59, 2011.

23 Schliekelman MJ, Creighton CJ, Baird BN, Chen Y, Banerjee P, Bota-Rabassedas N, Ahn Y-H, Roybal JD, Chen F, Zhang Y, Mishra DK, Kim MP, Liu X, Mino B, Villalobos P, RodriguezCanales J, Behrens C, Wistuba II, Hanash SM and Kurie JM: Thy-1+ cancer-associated fibroblasts adversely impact lung cancer prognosis. Sci Rep 7: 6478, 2017.

24 Zhu GC, Gao L, He J, Long Y, Liao S, Wang H, Li XX, Yi W, Pei Z, Wu M, Xiang J, Peng S, Ma J, Zhou M, Zeng Z, Xiang B, Xiong W, Tang K, Cao L, Li XX, Li G and Zhou Y: CD90 is upregulated in gastric cancer tissues and inhibits gastric cancer cell apoptosis by modulating the expression level of SPARC protein. Oncol Rep 34: 2497-2506, 2015.

25 Kumar A, Bhanja A, Bhattacharyya J and Jaganathan BG: Multiple roles of CD90 in cancer. Tumour Biol 37: 11611-11622, 2016
26 Romero D, O’Neill C, Terzic A, Contois L, Young K, Conley BA, Bergan RC, Brooks PC and Vary CPH: Endoglin regulates cancer-stromal cell interactions in prostate tumors. Cancer Res 71: 3482-3493, 2011.

27 Shahar T, Rozovski U, Hess KR, Hossain A, Gumin J, Gao F, Fuller GN, Goodman L, Sulman EP and Lang FF: Percentage of mesenchymal stem cells in high-grade glioma tumor samples correlates with patient survival. Neuro Oncol 19: 660-668, 2017.

28 Lee SB, Shim S, Kim MJ, Shin HY, Jang WS, Lee SJ, Jin YW, Lee SS and Park S: Identification of a distinct subpopulation of fibroblasts from murine dermis: $\mathrm{CD} 73^{-} \mathrm{CD} 105^{+}$as potential marker of dermal fibroblasts subset with multipotency. Cell Biol Int 40: 1008-1016, 2016.

29 Fonsatti E and Maio M: Highlights on endoglin (CD105): From basic findings towards clinical applications in human cancer. $\mathrm{J}$ Transl Med 2: 18, 2004.

30 Dickson MC, Martin JS, Cousins FM, Kulkarni AB, Karlsson S and Akhurst RJ: Defective haematopoiesis and vasculogenesis in transforming growth factor-beta 1 knock out mice. Development 121: 1845-1854, 1995.

31 Yu JX, Zhang XT, Liao YQ, Zhang QY, Chen H, Lin M and Kumar S: Relationship between expression of CD105 and growth factors in malignant tumors of gastrointestinal and its significance. World J Gastroenterol 9: 2866-2869, 2003.
Received October 18, 2018

Revised November 14, 2018

Accepted November 20, 2018 